\title{
Near-infrared observations of OGLE classical and type II Cepheid variables in the LMC
}

\author{
A. Bhardwaj ${ }^{1}$, L. M. Macri ${ }^{2}$, S. M. Kanbur ${ }^{3}$, C.-C. Ngeow ${ }^{4}$ \\ and H. P. Singh ${ }^{1}$ \\ ${ }^{1}$ Department of Physics \& Astrophysics, University of Delhi, Delhi 110007, India. \\ email: anupam.bhardwajj@gmail.com \\ ${ }^{2}$ Mitchell Institute for Fundamental Physics \& Astronomy, Department of Physics \& \\ Astronomy, Texas A\&M University, College Station, TX 77843, USA \\ ${ }^{3}$ State University of New York, Oswego, New York 13126, USA. \\ ${ }^{4}$ Graduate Institute of Astronomy, National Central University, Jhongli 32001, Taiwan.
}

\begin{abstract}
We present results from the Large Magellanic Cloud Near-infrared Synoptic Survey (LMCNISS) for classical and type II Cepheid variables that were identified by the Optical Gravitational Lensing Experiment (OGLE-III) catalogue. Multiwavelength time-series data for classical Cepheid variables are used to study light-curve structures as a function of period and wavelength. We exploit a sample of $\sim 1400$ classical and $\sim 80$ type II Cepheid variables to derive Period-Wesenheit relations that combine both optical and near-infrared data. The new PeriodLuminosity and Wesenheit relations are used to estimate distances to several Local Group galaxies (using classical Cepheids) and to Galactic globular clusters (using type II Cepheids). By appealing to a statistical framework, we find that fundamental-mode classical Cepheid PeriodLuminosity relations are non-linear around 10-18 days at optical and near-IR wavelengths. We also suggest that a non-linear relation provides a better constraint on the Cepheid PeriodLuminosity relation in type Ia Supernovae host galaxies, though it has a negligible effect on the systematic uncertainties affecting the local measurement of the Hubble constant.
\end{abstract}

Keywords. (stars: variables:) Cepheids - (galaxies:) Magellanic Clouds - (cosmology:) distance scale

\section{Introduction}

Cepheid variables are of vital importance for determining extragalactic distances, owing to the well-established Period-Luminosity relation (P-L or "Leavitt law", Leavitt \& Pickering 1912). That relationship allows one to derive an increasingly accurate and precise estimate of the Hubble constant (Freedman et al. 2001, Riess et al. 2016) independently of the cosmic microwave background by Planck (Ade et al. 2014).

The LMC has served as the first-rung of the cosmic distance ladder, mainly due to its close proximity and to the fact that it hosts a number of optically-identified Cepheid variables ( 4600, Soszyński et al. 2015). The distance to the LMC is also known with a precision of $\sim 2 \%$, when based on late-type eclipsing binary stars (Pietrzyński et al. 2013), thus allowing improved precision in the absolute calibration of the $\mathrm{P}-\mathrm{L}$ relations in the LMC. Over the past decade, near-IR observations of Cepheid variables have acquired a greater significance, because the $\mathrm{P}-\mathrm{L}$ relations are less sensitive to extinction and metallicity at longer wavelengths. However, the time-series $J H K_{s}$ observations of Cepheids in the LMC had been limited to a sample of $\sim 90$ stars in Persson et al.(2004), and most of the $\mathrm{P}-\mathrm{L}$ relations were based on single-epoch measurements, for example, Matsunaga, Feast \& Soszyński(2011), Inno et al.(2013), 

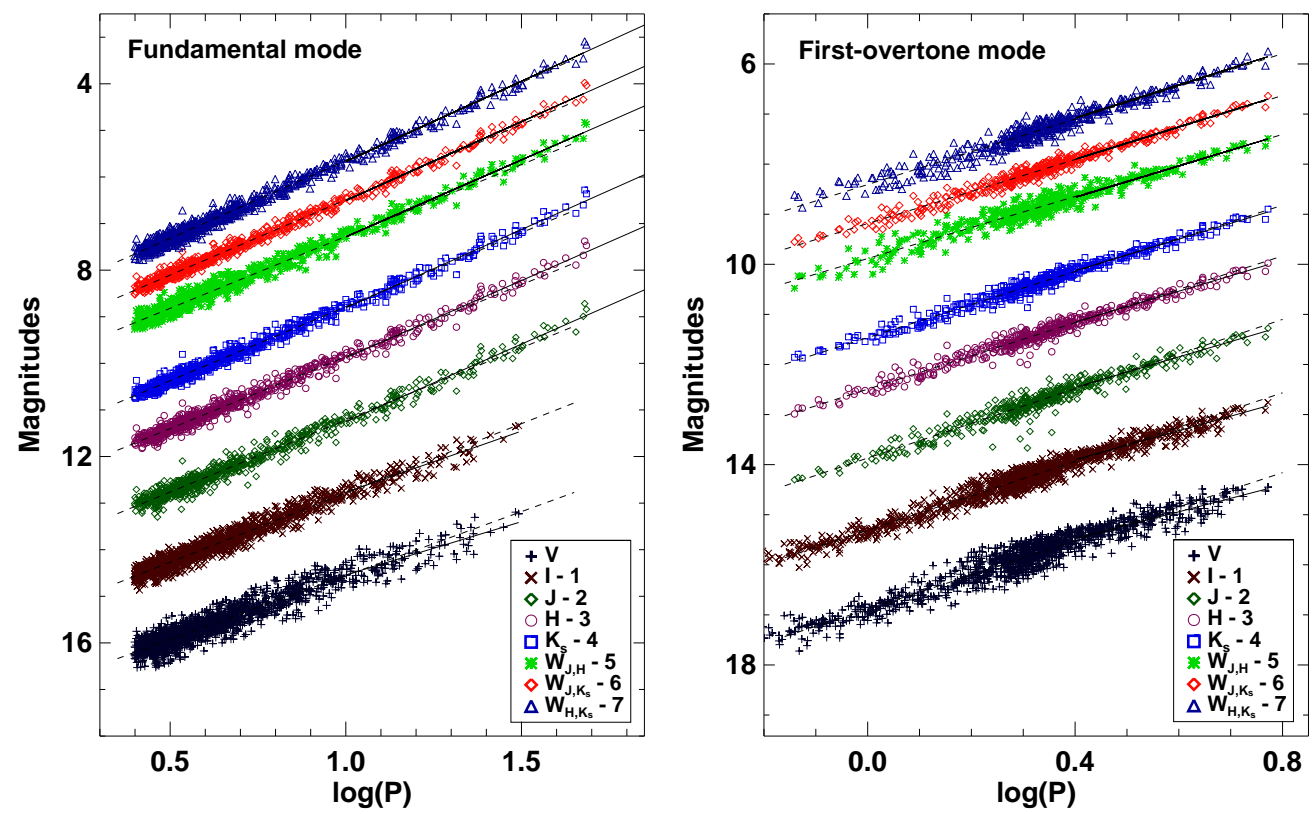

Figure 1. Multiband P-L relations and near-IR P-W relations for classical Cepheids in the LMC. The dashed and solid lines are best-fit regression lines over the entire, or the long-period, range respectively.

Macri et al.(2015) carried out a near-IR synoptic survey (LMCNISS) of the central $18^{\circ 2}$ of the LMC and cross-matched their catalogue with the OGLE-III survey. The authors identified $\sim 1500$ Cepheid variables with $J H K_{s}$ light-curves that had accurate periods and $V I$-band photometry from OGLE-III. The details regarding the data reduction, photometric accuracy, calibration into 2MASS photometric system, crowding and extinction corrections, etc., can be found in Macri et al. (2015, Paper I). In the following sections we summarize results based on the LMCNISS data, including a calibration of Period-Wesenheit $(\mathrm{P}-\mathrm{W})$ relations and their application to the distance scale (Paper II, Bhardwaj et al. 2016a), a statistical study of non-linearity in the Leavitt law (Paper III, Bhardwaj et al. 2016b), and near-IR P-L relations for type II Cepheids in the LMC (Paper IV, Bhardwaj et al. 2017a).

\section{Classical Cepheids}

Bhardwaj et al.(2015) used multiband time-series data, from OGLE-III and LMCNISS, for classical Cepheid variables in the LMC, to perform a Fourier analysis (Simon \& Lee 1981) of their light-curves, and presented a variation of the light-curve parameters as a function of period and wavelength. Macri et al.(2015) provided absolute calibration of the $\mathrm{P}-\mathrm{L}$ relations for $\sim 800$ fundamental-mode and $\sim 500$ first-overtone mode classical Cepheids in the LMCNISS. The fundamental-mode Cepheid $\mathrm{P}-\mathrm{L}$ relations provide $10 \times$ better constraints on the slopes and zero-points than previous work based on timeseries $J H K_{s}$ data. Bhardwaj et al.(2016a) then extended that work in order to derive optical and near-IR P-W relations using LMCNISS and OGLE data. Figure 1 displays multiband $\mathrm{P}-\mathrm{L}$ relations and near-IR $\mathrm{P}-\mathrm{W}$ relations for classical Cepheids based on the OGLE-LMCNISS data. It is evident from Figure 1 that $W_{J, K_{s}}$ Wesenheit exhibits the least scatter for both fundamental-mode and first-overtone-mode Cepheids. The ab- 


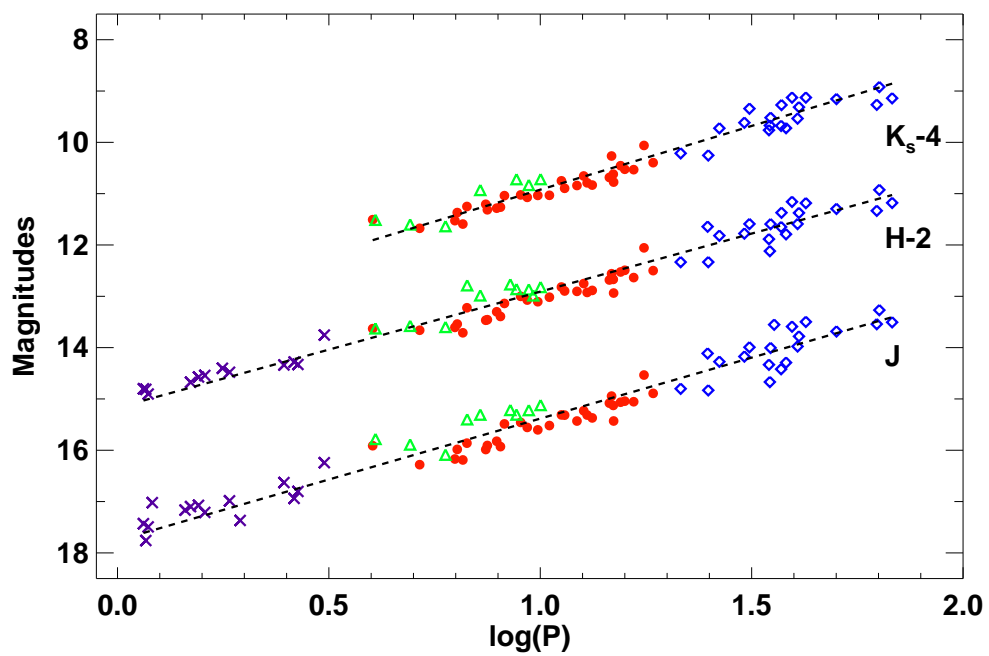

Figure 2. near-IR P-L relations for type II Cepheids in the LMC. The cross, circle, triangle and diamond symbols represent BL Herculis, W Virginis, Peculiar W Virginis and RV Tauri stars, respectively. The dashed line represents the best-fit linear regression over entire period range.

solute calibration based on the distance to the LMC derived from eclipsing binaries $(\mu=18.493 \pm 0.047)$ results in the following relation:

$W_{J, K_{s}}=-3.276 \pm 0.010-6.019 \pm 0.049 \quad(\sigma=0.077)$ (Fundamental)

$W_{J, K_{s}}=-3.216 \pm 0.024-6.518 \pm 0.049 \quad(\sigma=0.082)$ (First overtone),

where the zero-points of the $\mathrm{P}-\mathrm{L}$ relations are at 10 days. We used the calibration of LMC $W_{J, K_{s}}$ Wesenheit to estimate Cepheid-based distances to several Local Group galaxies covering a wide range of metallicity $(7.7<12+\log [O / H]<8.6 \mathrm{dex})$. We derived a global slope of $W_{J, K_{s}}$ Wesenheit, $-3.244 \pm 0.016 \mathrm{mag} \mathrm{dex}^{-1}$, and we did not find any significant metallicity dependence on $\mathrm{P}-\mathrm{W}$ relations. The Cepheid-based distance estimates are also found to be consistent with distances based on the tip of the red-giant branch.

We also developed a statistical framework that included the $F$-test, random-walk, testimator, segmented lines and the Davis test, to find possible statistically significant non-linearities in Cepheid P-L relations. Details of the statistics of the tests can be found in Bhardwaj et al.(2016b). We found that fundamental-mode Cepheid $\mathrm{P}-\mathrm{L}$ relations in the LMC exhibit a break at 10 days in optical bands and around 18 days in near-IR bands. The first-overtone-mode Cepheid $\mathrm{P}-\mathrm{L}$ relations were found to be non-linear at 2.5 days. These observed non-linearities can be attributed to sharp changes in the Fourier parameters at similar periods (Bhardwaj et al. 2015). Using LMCNISS data together with Cepheids in the type Ia supernovae host galaxies from Riess et al.(2011), we suggest that a non-linear version provides a twice better constraint on the slope and metallicity coefficients of the $\mathrm{P}-\mathrm{L}$ relations. The two-slope model was also adopted in the Cepheid $\mathrm{P}-$ $\mathrm{L}$ relations for the most precise $(2.4 \%)$ estimate of the local value of the Hubble constant by Riess et al.(2016).

\section{Type II Cepheids}

Bhardwaj et al.(2017a) used the $J H K_{s}$ observations of 81 type II Cepheid variables 
(16 BL Herculis, $31 \mathrm{~W}$ Virginis, 12 peculiar W Virginis and 22 RV Tauri) from OGLEIII and LMCNISS to derive $\mathrm{P}-\mathrm{L}$ and $\mathrm{P}-\mathrm{W}$ relations. Our near-IR $\mathrm{P}-\mathrm{L}$ relations, shown in Figure 2, are based on template-fitted mean magnitudes, where the templates are derived from $I K_{s}$-band data. We found that $\mathrm{P}-\mathrm{L}$ and $\mathrm{P}-\mathrm{W}$ relations are consistent with published results based on single-epoch data if long-period RV Tauri stars were excluded.

We used HST trigonometric parallaxes for $k$ Pav, and Gaia DR1 ones for VY Pyx, to estimate a distance to the LMC. In addition, the calibrated LMC P-L relations anchored to the distance drived from eclipsing-binary data yielded robust distances to 26 Galactic globular clusters given in Matsunaga et al.(2006). Those type II Cepheid-based distances are consistent with estimates using the $M_{V}-[\mathrm{Fe} / \mathrm{H}]$ relation for horizontal-branch stars. Bhardwaj et al.(2017b) also used the absolute calibration of type II Cepheid P-L relations in the LMC to estimate a distance to the Galactic centre, employing data from the Vista Variables in the Vía Láctea survey (Minniti et al. 2010).

\section{Conclusions}

We have presented a brief summary of near-IR observations of classical and type II Cepheid variables from the LMCNISS. The time-series data were used to derive new $\mathrm{P}-\mathrm{L}$ and Wesenheit relations for these variables with a greater sample-size and a better precision compared to results already in the literature. The LMCNISS data for Cepheids were also used as calibrators in the recent (and most precise) estimate of the Hubble constant based on Cepheid variables (Riess et al. 2016). The ongoing VISTA survey of the Magellanic Clouds (Cioni et al. 2011) is also providing near-IR data for Cepheid variables in the LMC, for example, Ripepi et al.(2012), Ripepi et al.(2015) but the timeseries photometry is limited to the $K_{s}$-band, in contrast to full-phased light-curves in LMCNISS.

\section{References}

Ade P. A. R. et al., 2014, A\&GA, 571, A16

Bhardwaj A. et al., 2016a, AJ, 151, 88

Bhardwaj A. et al., 2016b, MNRAS, 457, 1644

Bhardwaj A., Kanbur S. M., Singh H. P., Macri L. M., Ngeow C.-C., 2015, MNRAS, 447, 3342

Bhardwaj A. et al., 2017a, AJ, 153, 154

Bhardwaj A. et al., 2017b, $A \mathscr{G} A, 605$, A100

Cioni M.-R. L. et al., 2011, $A \& A, 527$, A116

Freedman W. L. et al., 2001, ApJ, 553, 47

Inno L. et al., 2013, $A p J, 764,84$

Leavitt H. S., Pickering E. C., 1912, Harvard College Observatory Circular, 173, 1

Macri L. M., Ngeow C.-C., Kanbur S. M., Mahzooni S., Smitka M. T., 2015, AJ, 149, 117

Matsunaga N., Feast M. W., Soszyński I., 2011, MNRAS, 413, 223

Matsunaga N. et al., 2006, MNRAS, 370, 1979

Minniti, D., Lucas, P. W., Emerson, J. P., et al. 2010, New Astronomy, 15, 433

Persson S. E. et al., 2004, AJ, 128, 2239

Pietrzyński G. et al., 2013, Nature, 495, 76

Riess A. G. et al., 2011, ApJ, 730, 119

Riess A. G. et al., 2016, ApJ, 826, 56

Ripepi V. et al., 2015, MNRAS, 446, 3034

Ripepi V. et al., 2012, MNRAS, 424, 1807

Simon N. R., Lee A. S., 1981, ApJ, 248, 291

Soszyński I. et al., 2015, Acta Astron., 65, 297 